\title{
Arrivées et conséquences des premiers agriculteurs en Centre Bretagne, 5000-1800 av. notre ère
}

\section{Charles-Tanguy Le Roux}

\section{Q OpenEdition}

\section{Journals}

Édition électronique

URL : http://journals.openedition.org/rao/4398

DOI : $10.4000 /$ rao.4398

ISSN : $1775-3732$

Éditeur

Presses universitaires de Rennes

\section{Édition imprimée}

Date de publication : 31 décembre 2017

Pagination : 358-359

ISBN : 978-2-7535-7540-0

ISSN : 0767-709X

\section{Référence électronique}

Charles-Tanguy Le Roux, "Arrivées et conséquences des premiers agriculteurs en Centre Bretagne 5000-1800 av. notre ère ", Revue archéologique de l'Ouest [En ligne], 34 | 2017, mis en ligne le 13 juin 2018, consulté le 25 février 2021. URL : http://journals.openedition.org/rao/4398 ; DOl : https:// doi.org/10.4000/rao.4398 
de Mathieu (Calvados), situé à une dizaine de kilomètres de la mer et fouillé en 2011 (et non 2010) sous la direction de David Giazzon. Il s'agit de la mise au jour dans le fossé d'une enceinte semi circulaire d'un habitat, de huit amas coquilliers constitués très majoritairement de moules. L'étude malacologique effectuée sur ces amas ont permis la mise en évidence de 32 espèces d'invertébrés marins dont l'essentiel n'a pas fait l'objet d'une collecte intentionnelle dans un but alimentaire, contrairement aux moules qui constituent $98 \%$ du nombre de restes. La collecte, la consommation et le rejet de ces produits complètent l'étude présentée qui est une des premières effectuée sur le littoral de la Manche en France pour l'âge du Bronze.

Le dernier article s'intéresse au métal et plus précisément aux modalités de dépôts non funéraires à l'âge du Bronze. Muriel Mélin compare ainsi les dépôts enfouis en pleine terre à ceux volontairement abandonnés dans les eaux des fleuves, en s'intéressant aux rythmes et ampleurs de ces dépôts en fonction des fluctuations climatiques. Elle constate deux périodes de discontinuité communes dans cette pratique d'enfouissement ou d'immersion d'objets métalliques, entre le Bronze moyen 1 et le Bronze moyen 2 soit vers 1500 av. notre ère, puis à la transition Bronze final-premier âge du Fer vers 800 av. notre ère, périodes qui s'avèrent globalement synchrones avec le début de deux péjorations climatiques.

Ces six articles sont suivis par les résumés de six autres contributions de longueur différente (1 à 9 pages), la plus conséquente étant consacrée par Pierre Stéphan à la question de la possibilité ou non d'un régression marine au Bronze final en Bretagne et par extension - et intérêt - au reste du littoral de la Manche. Elle est suivie par l'étude anthracologique de Hélène Seignac et al. des sites bretons Bronze ancien et moyen de Plouedern Leslouc'h (Finistère) et Lannion (Côtes-d'Armor), d'un point d'information de Ginette Auxiette sur l'état des recherches archéozoologiques en Plaine de Caen, d'une contribution de Yvon Dréano sur le pêche et la consommation de poissons marins à l'âge du Bronze en Bretagne et de deux notes : l'une de Cyril Marcigny sur la construction des paysages et les comportements agraires du $\mathrm{III}^{e}$ au $\mathrm{I}^{\mathrm{er}}$ millénaire en Normandie et l'autre de Muriel Fily sur les analyses spatiales en archéologie à travers notamment le travail effectué dans le cadre d'une thèse de doctorat sur les monuments funéraires et les dépôts métalliques dans le paysage à l'âge du Bronze dans le centreouest de la Bretagne et le Finistère littoral.

La publication rapide des actes de ce séminaire archéologique de l'ouest (SAO) à la fin de l'année même de sa tenue, permet à la communauté scientifique de mesurer à travers divers exemples concrets et souvent novateurs, les capacités et compétences de jeunes chercheurs qui alimentent et stimulent l'évolution de la recherche sur l'âge du Bronze dans la continuité, la dynamique et l'esprit des travaux entrepris dans cette région par Jacques Briard.

Cet ouvrage devait stimuler la communauté des bronziers pour que soit maintenant rapidement édités les autres séminaires restés encore non publiés ainsi que les actes de la table ronde de Rouen 2005 sur la transition Bronze-Fer.

Marc TALON Ministère de la Culture

JaLlot R., 2017 - Arrivées et conséquences des premiers agriculteurs en Centre Bretagne, 5000-1800 av. notre ère, Saarbrücken, Éditions universitaires européennes (ISBN 978-3-639-62368-0), 115 p.

Ce petit volume est directement issu d'un mémoire de Master 2 préparé sous la direction de F. Giligny et soutenu en 2015 devant l'université Paris 1 (Panthéon-Sorbonne). "Directement » est bien le mot qui convient car un Mémoire universitaire (élaboré comme on sait dans le cadre de ce qui reste plus ou moins un cénacle doté de ses propres codes) possède ses contraintes, souvent moins aisées qu'il n'y paraît à transposer dans une véritable publication à destination d'un lectorat élargi (même s'il reste spécialisé). On ne s'étonnera donc pas d'avoir ici et là quelques difficultés à entrer dans le jeu car un certain nombre de présupposés restent implicites (et pas toujours aisés à reconstituer pour qui n'aurait pas suivi le cheminement de l'auteure depuis son Master 1). Cette publication à compte d'auteur est aussi une édition "économique " au plein sens du terme : maquettage et qualité de l'illustration y restent en effet assez sommaires pour des raisons hélas faciles à comprendre.

Ceci dit, il serait dommage de se laisser rebuter par la forme. À l'heure où tant de travaux universitaires passent à la trappe (ou s'enlisent dans d'interminables procédures éditoriales), commençons donc par féliciter Rosalie Jallot d'avoir osé braver tous les obstacles pour nous livrer au plus vite le fruit de son travail.

Comme dit dans le préambule de l'ouvrage, les études spatiales en archéologie (dont ce mémoire fait partie) sont encore à la recherche d'un véritable cadre méthodologique. Elles se placent en effet à l'interface entre de multiples approches, aussi bien humaines que matérielles; les 
gérer toutes sans tomber dans un " sac de nœuds » n'est pas chose facile et R. Jallot a su y parvenir. Le souhait principal de l'auteure apparait cependant clairement dans ce même préambule; présenter le fruit d'une première recherche en archéologie des paysages et des territoires, pour mieux discuter la teneur des raisonnements et, à partir du corpus des observations de terrain, aboutir à des conclusions qui, comme c'est légitime à ce stade, restent encore largement interrogatives.

Spatialement, ce travail concerne le "Centre Bretagne", plus précisément le quart sud-ouest de l'actuel département des Côtes-d'Armor plus une large bande au nord-ouest du Morbihan; en tout, quelque $3000 \mathrm{~km}^{2}$ situés grossomodo entre Carhaix et Loudéac d'Ouest en Est et allant de Bourbriac à Pontivy du Nord au Sud. Comme présenté en Partie I de l'ouvrage, c'est une région géologiquement complexe et géographiquement fort variée. Mais aujourd'hui, après tant de millénaires d'activités humaines intenses et multiples, les traces des premiers occupants étudiés par R. Jallot ne s'y lisent plus qu'en faibles palimpsestes, même s'ils restent parfois spectaculaires comme les mégalithes (et spécialement les menhirs, objets, avec les carrières d'extraction mises en évidence dans l'ouvrage, d'une attention toute particulière).

Chronologiquement, la période prise en compte va du début du Néolithique moyen au début du Bronze ancien, soit de 4600 à 1800 avant notre ère. Ces quelque vingthuit siècles ont vu bien des changements : humainement comme matériellement, le monde des "petits princes de l'âge du Bronze» (pour reprendre l'expression de J. Briard) n'avait plus que des rapports bien ténus avec celui des pre- miers bâtisseurs de mégalithes. Suivre cette évolution à partir de la documentation disponible pour une région qui fut longtemps négligée des chercheurs supposait un considérable investissement sur le terrain en complément. C'est là une double gageure que R. Jallot a su relever avec panache, même si toutes ses conclusions n'emportent pas une adhésion sans faille.

De tout ce travail, la partie la plus marquante à nos yeux est sans doute le " cadre conceptuel » (\$1.3, p. 21-23), avec ses deux volets : « environnement naturel et paysage » d'une part, " perception d'un territoire» de l'autre. Ces notions sont exposées avec une maturité remarquable par cette jeune chercheuse, alors que trop de préhistoriens français se cantonnent dans cette " archéographie " du premier degré que fustigeait déjà P.-R. Giot en son temps. Tous les développements de l'ouvrage ne sont cependant pas aussi clairs et certains sont même difficiles à suivre, parfois desservis par une expression écrite qui reste à peaufiner. Parfois aussi, le piège $\mathrm{du}$ " raisonnement circulaire " n'est pas très loin, tandis que certains passages donnent, peut-être du fait d'une trop grande concision, l'impression d'avoir quelque peu « sollicité " les données.

Mais plutôt que d'en épingler les inévitables imperfections, saluons ici une " œuvre de jeunesse " prometteuse. Espérons surtout que, malgré la difficulté des temps pour les jeunes chercheurs, Rosalie Jallot puisse rapidement trouver l'opportunité de développer son travail, notamment dans le cadre d'une thèse de doctorat que nous attendons avec impatience.

Charles-Tanguy Le Roux

Collectif, 2016 - Potiers de Ger; l'aventure d'une industrie rurale du Moyen Âge au XXe siècle, Saint-Lô/Bayeux, Conseil départemental de la Manche - OREP, 2016.

Au XIV siècle, des potiers découvrent les extraordinaires capacités des argiles à grès qui chauffées à $1300{ }^{\circ} \mathrm{C}$ deviennent totalement imperméables. C'est le départ d'une production qui va vite s'étendre dans trois grands secteurs en Normandie : le Pays de Bray, le Cotentin/Bessin et le Domfrontais. Loin des pichets très décorés qui égayent les tables des seigneurs et bourgeois, les poteries de grès sont des vases utilitaires qui sont destinées à deux fonctions principales : le stockage et le transport du beurre et le stockage des salaisons. Dès lors commence d'une part l'orientation d'une partie importante de l'agriculture normande et bretonne vers la production de beurre et son exportation et d'autre part la généralisation des salaisons dans des grands récipients de grès. Les beurres bretons, alimentant pour une part la Marine verront les grès normands se diffuser sur nombre de territoires, parmi lesquels le Nouveau Monde. Plusieurs autres formes se développent dès le début, des pichets certes, mais surtout des récipients liés au travail du lait : jarres, pots à crémer, barattes et moules à fromage et plus tardivement les bouteilles. Cette poterie dont le principal défaut est de très mal tenir la chaleur a décliné bien des formes permettant la conservation des liquides. Plus tardivement, une production plus luxueuse verra le jour avec les fontaines très décorées et personnalisées, modeste dévoiement de cette céramique qui reste principalement utilitaire. 